\title{
Grupo terapêutico como proposta de acolhimento e redução do sofrimento psíquico
}

\section{nas universidades}

\author{
Therapeutic group as a proposal for reception and reduction of psychic suffering in universities \\ El grupo terapéutico como propuesta de acogida y reducción del sufrimiento psíquico en las \\ universidades
}

Recebido: 07/03/2021 | Revisado: 14/03/2021 | Aceito: 17/03/2021 | Publicado: 23/03/2021

Karen Silva de Castro

ORCID: https://orcid.org/0000-0001-8463-0322 Universidade do Estado do Pará, Brasil E-mail: silvakaren2021@gmail.com

Lauany Silva de Medeiros

ORCID: https://orcid.org/0000-0002-5683-6347 Universidade do Estado do Pará, Brasil E-mail: lauanymedeiiros@gmail.com

Michele Pinheiro Ferreira

ORCID: https://orcid.org/0000-0001-5316-9908 Universidade do Estado do Pará, Brasil

E-mail: michelepinheiroferreira@gmail.com

Nayara Fernanda Alves Moreira

ORCID: https://orcid.org/0000-0003-1094-7399 Universidade do Estado do Pará, Brasil E-mail: nfernandamoreira@gmail.com

Aline Ouriques de Gouveia

ORCID: https://orcid.org/0000-0001-8743-1433 Faculdade de Teologia, Filosofia e Ciências Humanas Gamaliel, Brasil

E-mail: aline_tuc@hotmail.com

Laís Araújo Tavares Silva

ORCID: https://orcid.org/0000-0002-6517-3338 Faculdade de Teologia, Filosofia e Ciências Humanas Gamaliel, Brasil

E-mail: laisaraujots@gmail.com

Carmen Lúcia de Araújo Paes

ORCID: https://orcid.org/0000-0001-9365-2451 Fundação Santa Casa de Misericórdia do Pará, Brasil E-mail: carmenaraujopaes@gmail.com

Valeria Regina Cavalcante dos Santos

ORCID: https://orcid.org/0000-0002-1264-8125 Fundação Santa Casa de Misericórdia do Pará, Brasil E-mail: valregsantos@hotmail.com

Amanda Ouriques de Gouveia

ORCID: https://orcid.org/0000-0002-6874-8352

Fundação Santa Casa de Misericórdia do Pará, Brasil

E-mail: mandinhaouriques@ hotmail.com

\begin{abstract}
Resumo
Descrever e caracterizar a população de estudantes observada em um encontro de grupo terapêutico, realizado por uma universidade pública no estado do Pará. Trata-se de um relato de experiência de cunho qualitativo, exploratório e descritivo, de caráter crítico-reflexivo que engloba os conceitos do matriciamento em saúde mental, visando outorgar um suporte psicológico para a comunidade acadêmica. Durante a apresentação do projeto "Acolhidamente", no início do ano letivo de 2020, buscou-se integrar atividades complementares educativas e de conversa com os acadêmicos dos cursos de enfermagem, educação física, fisioterapia e ciências biológicas. O Grupo Terapêutico fundamentou o sofrimento psíquico em uma roda de conversa, que servisse como dispositivo psicoterapêutico na prevenção do sofrimento psíquico, e consequentemente, na melhor promoção do bem-estar psicológico. O estudante universitário enfrenta uma variedade de condições de risco para sua saúde mental e bem-estar, assim com os resultados obtidos na aplicação da atividade educativa e métodos, foi possível identificar e discutir os sinais e sintomas indicadores de estresse, ansiedade, depressão e burnout entre os estudantes.
\end{abstract}

Palavras-chave: Saúde mental; Terapia; Universidade. 


\begin{abstract}
Describe and characterize the student population observed at a therapeutic group meeting, held by a public university in the state of Pará. This is a qualitative, exploratory and descriptive experience report, of a critical-reflective nature that encompasses the concepts matriculation in mental health, aiming to provide psychological support to the academic community. During the presentation of the "Acolhecido" project, at the beginning of the 2020 academic year, we sought to integrate complementary educational activities and conversation with academics from the nursing, physical education, physiotherapy and biological sciences courses. The Therapeutic Group grounded psychological suffering in a conversation circle, which served as a psychotherapeutic device in the prevention of psychological suffering, and consequently, in the best promotion of psychological well-being. The university student faces a variety of risk conditions for his mental health and well-being, as well as the results obtained in the application of the educational activity and methods, it was possible to identify and discuss the signs and symptoms indicating stress, anxiety, depression and burnout among students.
\end{abstract}

Keywords: Mental health; Therapy; University.

\title{
Resumen
}

Describir y caracterizar la población estudiantil observada en una reunión de grupo terapéutico, realizada por una universidad pública del estado de Pará. Se trata de un relato vivencial cualitativo, exploratorio y descriptivo, de carácter crítico-reflexivo que engloba los conceptos matriculación en salud mental, con el objetivo de brindar apoyo psicológico a la comunidad académica. Durante la presentación del proyecto "Acolhecido", al inicio del curso 2020, se buscó integrar actividades educativas complementarias y conversación con académicos de los cursos de enfermería, educación física, fisioterapia y ciencias biológicas. El Grupo Terapéutico fundamentó el sufrimiento psicológico en un círculo de conversación, que sirvió como dispositivo psicoterapéutico en la prevención del sufrimiento psicológico y, en consecuencia, en la mejor promoción del bienestar psicológico. El universitario enfrenta una variedad de condiciones de riesgo para su salud y bienestar mental, así como los resultados obtenidos en la aplicación de la actividad y métodos educativos, fue posible identificar y discutir los signos y síntomas indicativos de estrés, ansiedad, depresión y agotamiento entre los estudiantes.

Palabras clave: Salud mental; Terapia; Universidad.

\section{Introdução}

A transição do Ensino Médio para o Ensino Superior coincide com um período crucial do desenvolvimento humano, que é a adolescência, caracterizado por uma revolução bio-psicossocial. A marca desse período é a passagem do estado infantil para o de adulto e as características psicológicas desse movimento evolutivo, sua expressividade e manifestações em nível de comportamento e de adaptação social dependem da cultura e da sociedade na qual o processo da adolescência se desenvolve (Cerchiari, 2015).

Nesse sentido, o conflito do tornar-se adulto ocorre em paralelo ao luto pela perda da estrutura infantil. Ademais, os adolescentes que têm a oportunidade de frequentar uma escola com o objetivo de fazer um curso universitário precisam definir, por imposição do sistema educacional vigente, aos 16 anos de idade, o que desejam ser no futuro, justamente no auge de sua crise de identidade (Levinsky, 1998).

Para uma parcela significativa dos jovens brasileiros esse processo é vivenciado na faixa etária de 17 e 19 anos, ao mesmo tempo em que, ao ingressarem na Universidade, têm que lidar com a adaptação acadêmica. Assim, essas vivências poderão desencadear situações de crises que se manifestam através de depressões, alcoolismo, evasão escolar, dificuldades de aprendizagem, relacionamentos pessoais insatisfatórios, ligações de amizades prejudiciais e isolamento (Fernandes \& Rodriges, 1995).

$\mathrm{Na}$ Universidade o processo ensino-aprendizagem pode ser comprometido pela estratégia de ensino, relação professor-aluno, formas de avaliação, e deficiências nas instalações físicas e de materiais das instituições. Essas circunstâncias, na vida do jovem, somadas ao precisar sair da casa dos pais, separar-se dos amigos e de um círculo seguro e habitual de relacionamentos, pode desencadear situações de crise e, até, doenças (Jorge \& Rodrigues, 1995).

De acordo com Loreto (1985): 
Problemas psicossociais, tais como ansiedade, depressão, preocupações com os estudos e dificuldades de relacionamentos, são comumente encontrados em estudantes universitários e, quando não avaliados e tratados adequadamente, podem levar às evasões que são onerosas para o ensino público, para a sociedade e, principalmente, para o próprio estudante (Loreto, 1985).

Dessa forma, destaca-se o acolhimento dos discentes e um grupo terapêutico, construído e idealizado conforme as necessidades de saúde/doença e realidade social encontrada. A partir disso, essa estratégia surge a fim de possibilitar aos alunos um lugar de fala, expressão, segurança e escuta segura, além de avanço na prevenção de sofrimentos psíquicos e promoção do bem-estar mental, pois exerce o papel de um dispositivo construtor do paradigma social (Osse, 2013).

Este estudo pretendeu descrever e caracterizar a população de estudantes observada em um encontro de grupo terapêutico, realizado por uma universidade pública no estado do Pará, destacando a nova possibilidade de pesquisas e contribuições com sugestões imediatas, de médio e longo prazo, na assistência em saúde mental de todos os alunos envolvidos.

\section{Metodologia}

Trata-se de um relato de experiência de cunho qualitativo, exploratório e descritivo, de caráter crítico-reflexivo que engloba os conceitos do matriciamento em saúde mental, visando outorgar um suporte psicológico para a comunidade acadêmica para que assim o docente e/ou a faculdade transitem de sua real condição de "ensinar" à condição de ser um profissional ou centro de ensino atuante e competente, fortalecendo o vínculo da universidade com a comunidade, reafirmando o seu papel na sociedade. (Ministério da Saúde, 2011)

Logo, tal estudo caracterizou-se por uma ação de educação em saúde em forma de grupo terapêutico desenvolvido no município de Tucuruí, localizada na região sudeste do estado do Pará, com acadêmicos calouros recém aprovados na Universidade do Estado do Pará, campus XIII, sobre as perspectivas e as principais dúvidas no que se refere ao ensino superior.

Desse modo, a educação permanente em questão foi desenvolvida, pela coordenação do campus, docentes especialistas em saúde mental e veteranos dos cursos ofertados pela universidade em parceria com o projeto "Acolhidamente", o qual aborda o sofrimento psíquico junto aos acadêmicos dos cursos de enfermagem, educação física, fisioterapia e ciências biológicas, por meio de roda de conversa como dispositivo psicoterapêutico na prevenção do sofrimento psíquico e promoção do bem-estar psicológico.

A ação ocorreu no início do ano letivo de 2020, sendo que foram estipulados os seguintes critérios de inclusão para a participação na roda de conversa: alunos devidamente ligados a Universidade do Estado do Pará, preferencialmente, calouros dos cursos ofertados pela instituição e que possuísse disponibilidade para a participar do grupo terapêutico. Já como critérios de exclusão, incluiu acadêmicos ligados a rede privada de ensino, os que foram matriculados posteriormente a ação e os que optassem por não participar. Desse modo, obedecendo os parâmetros de inclusão e exclusão, participaram do grupo terapêutico cerca de 25 acadêmicos da rede pública de ensino.

Logo, é importante ressaltar que o grupo terapêutico foi desenvolvido por meio do método da problematização com a aplicação das 5 etapas do Arco de Maguerez (Esquema 1), sendo a primeira a observação da realidade que foi realizada ao constatar importância de ações com a temática de saúde mental nos centros de ensino, principalmente, no ensino superior, haja a vista, que o ingresso nesta fase acadêmica é uma transição que traz potenciais repercussões para o desenvolvimento psicológico dos jovens, pois é marcada por processos complexos de transição e adaptação, além de conflitos e questões novas a nível pessoal. 


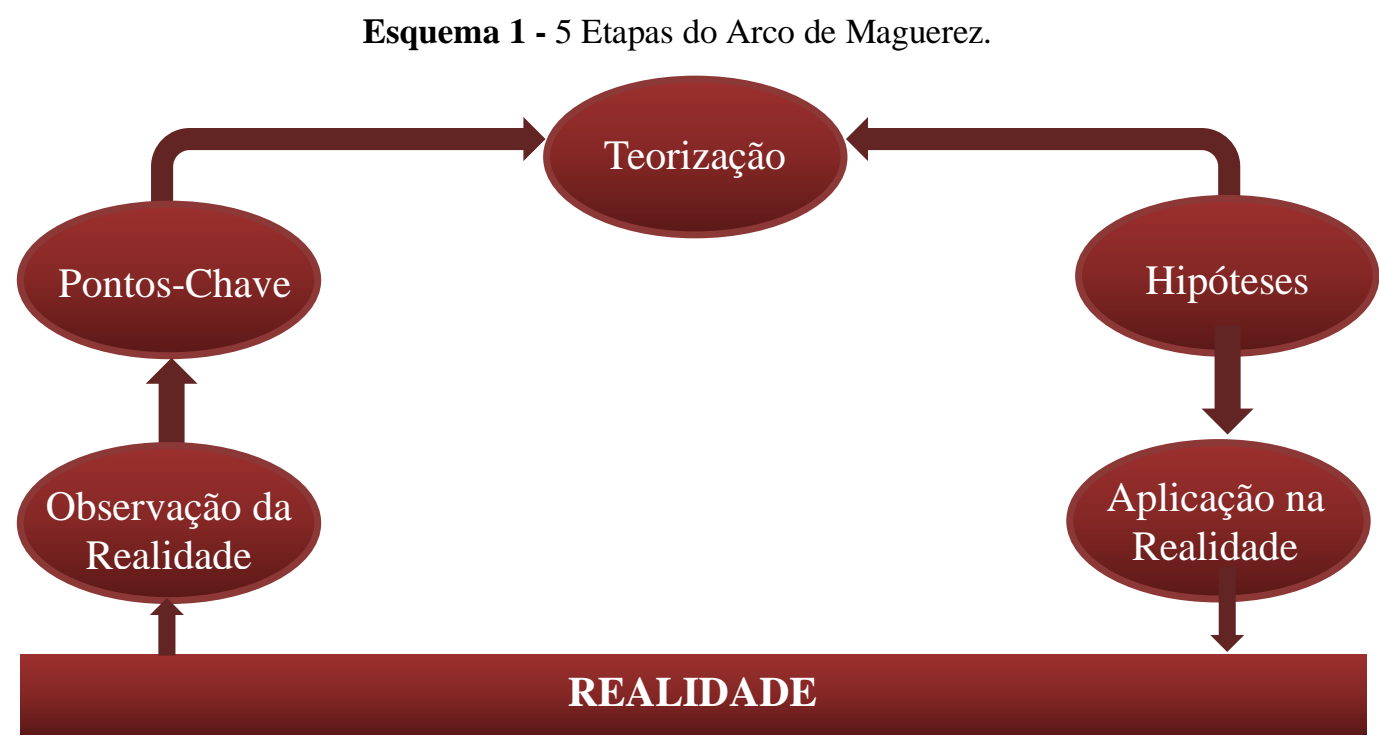

Fonte: Autores (2020).

Com isso, foi reconhecido a necessidade de fazer o acolhimento dos estudantes calouros e assim começou a segunda fase com a retirada dos pontos-chave da problemática e seus principais determinantes, considerando: Quais são principais variáveis que interferem no processo de adaptação do estudante? Quais são as expectativas do estudante para a área escolhida e para a universidade? Como amenizar a problemática no meio acadêmico?

Nesse contexto, a fim de subsidiar o conhecimento acerca da problemática e assim subsidiar o restante das etapas, foi necessário expandir o entendimento no que concerne a saúde mental dos jovens nos centros de ensino, para tal, fez-se necessário realizar uma a revisão bibliográfica através das plataformas de busca PubMed, Lilacs, BVS e Scielo, onde utilizouse os descritores "assistência à saúde mental”, "jovens", "serviços escolares de saúde mental”, e "educação em saúde".

A partir disso ocorreu o estabelecimento das hipóteses para solução do problema, que foram baseados no "Guia Prático de Matriciamento Saúde Mental" oferecido pelo Ministério da Saúde, o qual apresenta o modo de como se deve fazer um projeto terapêutico singular, nesse viés, tal método constituiu um plano de ação viável para a comunidade acadêmica, pois baseia-se na premissa de que nas práticas de saúde coletiva é fundamental levar em consideração não só o indivíduo, mas todo o seu contexto social.

Por fim, ocorreu a fase de aplicação na realidade que foi realizada em forma de grupo terapêutico com os acadêmicos que aconteceu utilizando diversas metodologias em formato aberto e técnicas de dinâmicas em grupo, acompanhadas de reflexões entre os participantes acerca de suas dificuldades, percepções e sentimentos.

\section{Resultados}

Durante a apresentação do projeto "Acolhidamente", criado pela Universidade do Estado do Pará (UEPA) no município de Tucuruí-PA, que ocorreu no início do ano letivo de 2020, buscou-se integrar atividades complementares educativas e de conversa com os acadêmicos dos cursos de enfermagem, educação física, fisioterapia e ciências biológicas, que frequentam o campus XIII, visando abordar sobre o sofrimento psíquico e suas dimensões.

Nesse sentido, o Grupo Terapêutico almejou fundamentar o tema em questão, por meio de uma roda de conversa, que servisse como dispositivo psicoterapêutico na prevenção do sofrimento psíquico, e consequentemente, na melhor promoção do bem-estar psicológico. A partir disso, a ação contou com o apoio de docentes, enfermeiros especialistas em saúde mental e discentes de séries anteriores, para a elaboração e prática da temática proposta, somando ao todo 25 encontros, com tempo de duração entre 20 a 45 minutos cada. 
Além disso, foram promovidas atividades complementares de lazer, as quais se tornaram essenciais para na realização do projeto, no que se refere a criação de vínculos e afinidade entre os participantes para que desse modo, eles compartilhassem suas vivências, medos, aspirações e objetivos, de maneira que promovesse maior interrelação entre os acadêmicos das demais graduações.

Dessa forma, foi proposta a separação de subgrupos de indivíduos, sendo eles relacionados aos acadêmicos que acabam de ingressar na faculdade e os veteranos, para melhor fundamentação dos eixos temáticos, objetivando conhecer e reconhecer os pontos de vista e vivências dos participantes e assim, facilitar o entendimento do tema de acordo com a necessidades identificadas no público-alvo. Em seguida, os docentes e especialistas de saúde mental abordavam sobre a problemática, para melhor entendimento do assunto.

Nessa conjectura, o grupo terapêutico ocorreu na quadra poliesportiva da instituição, para maior lazer e agregação de pessoas, sendo iniciado com uma música de boas-vindas aos acadêmicos, por meio do som de violão em uníssono. Por conseguinte, deu-se início a identificação de cada aluno, buscando maior familiaridade entres os mesmos, e em continuidade, buscou-se elencar o tema abordado para que todos tivessem conhecimento da temática.

Mediante a isso, foi dado abertura para o debate sobre as observações de cada discente, sendo incialmente realizado pelos calouros, os quais abordaram sobre suas expectativas na área escolhida, bem como se foi a primeira opção de curso, os anseios, perspectiva do campus e principais dúvidas nessa nova etapa. Desse modo, observou-se que a maioria se encontrava comedida em relação ao novo momento de suas vidas, e ao mesmo tempo, ansiosos pelo próximo passo em sua carreira.

Além do mais, considerou-se a pauta de dúvidas em relação a nova etapa, onde foram identificadas perguntas quanto a complexidade do curso, carga-horária e avaliação das disciplinas, sendo elas sanadas pelos discentes veteranos e docentes do curso, para minimizar a ansiedade e apreensão quanto a graduação. Outrora, foi dimensionado sobre as possíveis dificuldades que poderão encontrar durante sua formação, enfatizando que o corpo docente e da instituição estão contribuindo no auxilio de suas necessidades biológicas e emocionais, como por exemplo, o apoio do grupo terapêutico.

Outrossim, deu-se sequência ao outro subgrupo referente aos veteranos da instituição, os quais relataram as experiências vividas desde a aprovação no vestibular até o atual semestre, pautando as apreensões no contexto universitário que afetam a vida pessoal e profissional, referindo suas prováveis intempéries. Subsequente a isso, foram amplamente enfatizadas as necessidades de se adaptar ao cenário, e cada vez mais se padronizar ao curso, de forma que facilite a organização do tempo acadêmico com o lazer pessoal.

Consoante a isso, os mediadores do projeto buscaram intervir e elencar atividades de debate aberto, para possibilitar outras dinâmicas em grupo, como cantar músicas conhecidas que trouxessem sentimento de alegria, e atividades reflexivas entre os participantes, com o intuito de reconhecer e assimilar as dificuldades encontradas, as percepções e sentimentos individuais quanto as exposições de vivências de cada um, além de oferecer auxilio e escuta às estimas dos acadêmicos.

Ao fim do projeto, foi realizado um abraço coletivo, que confraternizou com as emoções de cada participante, e promoveu um ambiente mais solícito aos acadêmicos que adentravam a instituição. Sendo assim, a orientação terapêutica consolidou seu objetivo a partir do acolhimento do público-alvo, oferecendo uma escuta segura, apoio emocional e atenção para alivio de sintomas, os quais encontravam-se bastante evidentes em relação a apreensão do desconhecido e ansiedade sobre o novo.

Nessa perspectiva, considera-se que essa assistência terapêutica seja essencial para minimizar o sofrimento psíquico dos graduandos, observando suas particularidades e observações, e assim, planejando uma conduta segura e íntegra sobre as necessidades de cada indivíduo analisado. 


\section{Discussão}

\section{Dificuldades na transição para a universidade}

A universidade é caraterizada como um espaço fundamental para o desenvolvimento de vida do ser humano, pois proporciona o fortalecimento e a extensão de seu repertório, competências e habilidades profissionais e pessoais, da mesma forma em que promove um aperfeiçoamento de seu funcionamento cognitivo, ratificando-se como um período gerador de processos positivos na vida de seus acadêmicos. No entanto, por ser um período de transição e mudanças, proporcionando novas demandas e exigindo uma adaptação abrupta dos estudantes em um curto espaço de tempo, podem ocorrer casos de estresse que interferem diretamente na saúde física e mental dos alunos.

A princípio, vale ressaltar que a introdução à instituição de ensino superior gera consideráveis transformações na vida dos indivíduos. Nesse sentido, o discente durante a iniciação de sua vivência acadêmica depara-se com um conjunto de desafios institucionais, interpessoais, pessoais e familiares. A partir disso, ocorrem situações comuns desse período, que são impostas pelo Ensino Superior (ES), que constituem em importantes estressores para os universitários, como: exigências sociais de maior autonomia, necessidade de constante aprovação, dificuldade em administrar o seu tempo de maneira ideal, saída do conforto de casa, em alguns casos, problemas em lidar com a ausência de afeto, mudanças nas redes de amizade e cobrança por bom desempenho acadêmico (Dias, et al 2019).

Dessa forma, a adaptação acadêmica necessita de uma sequência de condições que permitem o sentimento de integração do calouro ao curso e à instituição. Ou seja, a adequação ao ES percorre desde a consciência a respeito das oportunidades ofertadas pela universidade e a sensação de pertencimento a turma até a rede de apoio, em que o recémuniversitário pode e deve recorrer em situações de dificuldades.

Ademais, é comum evidenciar que a inserção no curso superior se suceda com a separação da família corroborando negativamente para que o processo de adaptação ocorra. Nessa perspectiva, compreende-se que a saudade de casa tem sido constantemente identificada como um efeito desfavorável à necessária ação de se ajustar em um novo paradigma, que no caso é mudar-se da casa de seus pais para morar em outra cidade sozinho ou com outras pessoas.

De Oliveira e Dias (2014), em seus estudos evidenciaram que os estudantes que residem sem a família apresentam maior possibilidade de desenvolver níveis mais elevados de estresse em relação aos que convivem com seus familiares, sendo que esse processo de estresse acadêmico se refere a uma reação adaptativa ao meio em que foi inserido, que se prolongados podem resultar em diversos problemas, como desempenho acadêmico pobre.

Com isso, proporcionar um ambiente confortável e acolhedor é essencial para fornecer ao acadêmico segurança e liberdade de expressar seus medos e aflições, pois esse é um período caracterizado pelo alto índice de desenvolvimento de transtornos mentais em jovens. Segundo Ariño e Bardagi (2018), a população universitária está vulnerável ao desenvolvimento de alguns transtornos mentais, como por exemplo, a depressão, a ansiedade e o stress, sendo apresentada uma prevalência elevada de 15 a 25\% dos universitários que irão apresentar algum transtorno mental durante sua formação.

Alguns estudos epidemiológicos e de prevalência indicam que a presença de transtornos mentais não psicóticos neste público é significantemente maior que na população geral e em adultos jovens não universitários. A partir destes indicadores, a hipótese é de que aspectos relacionados à vida acadêmica e à carreira podem impactar na saúde mental desta população e explicar, parcialmente, a alta prevalência de transtornos mentais na mesma (Ariño \& Bardagi, 2018, p.44).

\section{Grupo terapêutico com foco na adaptação acadêmica}

O grupo terapêutico é uma forma de terapia em que um grupo de indivíduos, que apresentam características em comum ou enfrentam os meus desafios, reúnem-se regularmente com o objetivo de proporcionar que os participantes, na 
condição de co-terapeutas, consigam se encaixar em situações parecidas ou distintas de outros participantes, interatuando a partir de suas percepções e sensações, o que amplifica a sua compreensão de si e também a autoestima interligada ao sentimento de pertencimento (Couto \& Vicente, 2018).

Nesse viés, a terapia em grupo é uma importante estratégia de promoção da saúde do estudante, pois, ao potencializar as trocas dialógicas e o comportamento de experiências, melhora a capacidade adaptativa e transformativa dos envolvidos. Logo, sua finalidade é possibilitar um espaço de escuta e acolhimento aos estudantes, de modo que posso auxiliá-los a lidar com a ansiedade na vida acadêmica e construir estratégias de prevenção, proteção e cuidado a saúde mental.

De acordo com Scherer et al. (2017) em sua pesquisa sobre “. Grupoterapia com estudantes de enfermagem durante a transição teórico-prática", apontou que:

O grupo terapêutico favorece a exploração da subjetividade ao atuar como um "laboratório social" no qual os participantes reproduzem os papéis que ocupam no seu cotidiano. Quando o grupo é constituído por uma população específica, com características semelhantes, há uma facilitação para a identificação (Scherer et al, 2017).

A partir disso, nota-se que ao longo do percurso da vida, os indivíduos estabelecem diversos vínculos grupais, o que proporciona um fator favorável ao estabelecimento do grupo como um recurso terapêutico que pode auxiliar o estudante a fazer contato com conteúdo internos, funções sociais desempenhadas por si, comportamentos e expectativas em relação à universidade e diferentes percepções mediante as dificuldades na convivência com outros colegas e professores.

Nesse sentido, a iniciativa de formação do grupo terapêutico é fundamental para auxiliar esse processo de transição do universitário, uma vez que, o grupo funciona como um espaço de trocas, que é apto de espelhar para cada participante aquilo que o próprio ainda não foi capaz de perceber em si, auxiliando, consequentemente, em sua autocompreensão. Tendo por base essa linha de raciocínio, infere-se que nos grupos terapêuticos, os participantes apoiam-se uns aos outros, compartilhando de angustias e desafios, além de encontrar pessoas com conflitos semelhantes, contribuindo para o processo de crescimento pessoal (Deniz \& Aires, 2018).

Além disso, torna-se imprescindível ressaltar que na terapia de grupo cada participante tem a possibilidade de executar o papel de agente terapêutico para com os demais, o que, por sua vez, viabiliza ao cliente autoconhecimento, apoio para enfrentar suas questões internas, transformações de consciência e conduta, restauração de sua saúde mental, atenuação de tensões, aperfeiçoamento das relações interpessoais, progresso pessoal e, logo, a ampliação de suas possibilidades de reorganização da própria vida.

Por fim, reitera-se que a inserção do estudante no contexto universitário é marcada por diversas mudanças e pela necessidade de adaptar-se ao novo, tendo em vista que, em sua maioria, esses universitários se deparam com a mudança de cidade, com a distância da família e dos amigos e a necessidade de se adaptar às regras da universidade e de estabelecer novos vínculos de amizade que, por sua vez, constituirão sua nova rede de apoio. E, para auxiliar esses jovens na resolução de conflitos e angustias advinda desse processo de mudanças, tem-se utilizado técnicas de teria em grupo que oferece aos alunos suporte psicológico, favorecido pelo contato com pares que enfrentam situações semelhantes no processo de adaptação acadêmica.

\section{Conclusão}

Pode-se concluir, à face do exposto nos tópicos deste trabalho, que o estudante universitário enfrenta uma variedade de condições de risco para sua saúde mental e bem-estar. A partir dos resultados obtidos com a aplicação da atividade educativa e métodos, foi possível identificar e discutir os sinais e sintomas indicadores de estresse, ansiedade, depressão e burnout entre os estudantes. 
Research, Society and Development, v. 10, n. 3, e51010313623, 2021

(CC BY 4.0) | ISSN 2525-3409 | DOI: http://dx.doi.org/10.33448/rsd-v10i3.13623

É evidente a vulnerabilidade que a população universitária se encontra desde o ingresso na universidade, sua trajetória acadêmica e formação do futuro profissional. Todas as fases da graduação e suas relações podem configurar como processos criadores ou patogênicos para o discente, afetando o mesmo em nível educativo, psicológico e social.

Portanto, toda a diversidade de fatores que favorecem o sofrimento psíquico e ferem i bem-estar dos estudantes devem ser objeto de reflexão e estudo aprofundado das universidades e seus gestores, os quais deves coordenar suas atividades para difundir e aprimorar as políticas de apoio psicológico ou psicopedagógico aos universitários.

\section{Referências}

Ariño, D. O., \& Bardagi, M. P. (2018). Relação entre fatores acadêmicos e a saúde mental de estudantes universitários. Revista psicologia em pesquisa, 12(3).

Castro, V. R. (2017). Reflexões sobre a saúde mental do estudante universitário: estudo empírico com estudantes de uma instituição pública de ensino superior. Revista gestão em foco, $9(1), 380-401$.

Cerchiari, E. A. N. (2015). Saúde mental e qualidade de vida em estudantes universitários.

Couto, D. B. D., \& Vicente, C. C. (2018). Psicoterapia de grupo con foco en la adaptación académica: un experimento con estudiantes. Estudos e Pesquisas em Psicologia, 18(3), 812-830.

Chiaverini, D. H., Gonçalves, D. A., Ballester, D., Tófoli, L. F., Chazan, L. F., Almeida, N., \& Fortes, S. (2011). Guia prático de matriciamento em saúde mental.

de Oliveira, C. T., \& Dias, A. C. G. (2014). Dificuldades na trajetória universitária e rede de apoio de calouros e formandos . Psico, 45 (2), 187-197.

Dias, A. C. G., Carlotto, R. C., Oliveira, C. T. D., \& Teixeira, M. A. P. (2019). Dificuldades percebidas na transição para a universidade. Revista brasileira de orientação profissional. São Paulo, SP. Vol. 20,n. 1 (jan./jun. 2019), p. 19-30.

Diniz, N. F. P. D. S., \& Aires, S. (2018). Grupo de escuta e reflexão com estudantes universitários. Vínculo, 15(1), 61-75.

Fernandez, J. M., \& Rodrigues, C. R. C. (1993). Estudo retrospectivo de uma populaçäo de estudantes de medicina atendidos no ambulatório de clínica psiquiátrica do Hospital das Clínicas da Faculdade de Medicina de Ribeiräo Preto. Medicina (Ribeiräo Preto), 258-69.

Jorge, M. S., Rodrigues, A. R. F. (1995). Serviço de apoio ao estudante oferecido pela escola de enfermagem no Brasil. Revista Latino-Americana de Enfermagem, 3(1) 59-68.

Levinsky, D. L. (1998). Adolescência: reflexões psicanalíticas. Casa do Psicólogo, 1(1): 10-15.

Loreto, G. (1985). Uma experiência de assistência psicológica e psiquiátrica a estudantes universitários. [Tese de Livre Docência]. Cento de Ciências da Saúde - Universidade Federal de Pernambuco, 8(2): 105-111.

Osse, C. M. C. (2013). Saúde mental de universitários e serviços de assistência estudantil: estudo multiaxial em uma universidade brasileira.

Scherer, Z. A. P., Scherer, E. A., \& Carvalho, A. M. P. (2017). Grupoterapia com estudantes de enfermagem durante a transição teórico-prática. Revista Latino-Americana de Enfermagem, 15(2).

Farinhuk, P. D. S., Savaris, L. E., Franco, R. S. (2021). Transtorno mental e sofrimento psíquico: representações sociais de profissionais da Atenção Básica à Saúde. Research, Society and Development, 10(3). 